\title{
Long-term risk of all-cause mortality in live kidney donors: a matched cohort study
}

\author{
Eunjeong Kang ${ }^{1}$, Sehoon Park ${ }^{2}$, Jina Park ${ }^{3}$, Yaerim Kim ${ }^{4}$, Minsu Park ${ }^{5}$, Kwangsoo Kim ${ }^{6}$, Hyo Jeong Kim ${ }^{7}$, \\ Miyeun $\mathrm{Han}^{8}$, Jang-Hee Cho ${ }^{9}$, Jung Pyo Lee ${ }^{10}$, Sik Lee ${ }^{11}$, Soo Wan Kim ${ }^{12}$, Sang Min Park ${ }^{13}$, Dong-Wan Chae ${ }^{14}$, \\ Ho Jun Chin ${ }^{14}$, Yong Chul Kim ${ }^{15}$, Yon Su Kim ${ }^{15}$, Insun Choi ${ }^{7}$, Hajeong Lee ${ }^{15}$ \\ For further information on the authors' affiliations, see Additional information.
}

Background: Long-term outcomes of live kidney donors remain controversial, although this information is crucial for selecting potential donors. Thus, this study compared the long-term risk of all-cause mortality between live kidney donors and healthy control.

Methods: We performed a retrospective cohort study including donors from seven tertiary hospitals in South Korea. Persons who underwent voluntary health screening were included as controls. We created a matched control group considering age, sex, era, body mass index, baseline hypertension, diabetes, estimated glomerular filtration rate, and dipstick albuminuria. The study outcome was progression to end-stage kidney disease (ESKD), and all-cause mortality as identified in the linked claims database.

Results: We screened 1,878 kidney donors and 78,115 health screening examinees from 2003 to 2016. After matching, 1,701 persons remained in each group. The median age of the matched study subjects was 44 years, and $46.6 \%$ were male. Among the study subjects, $2.7 \%$ and $16.6 \%$ had underlying diabetes and hypertension, respectively. There were no ESKD events in the matched donor and control groups. There were $24(1.4 \%)$ and 12 mortality cases $(0.7 \%)$ in the matched donor and control groups, respectively. In the age-sex adjusted model, the risk for all-cause mortality was significantly higher in the donor group than in the control group. However, the significance was not retained after socioeconomic status was included as a covariate (adjusted hazard ratio, 1.82; 95\% confidence interval, 0.87-3.80).

Conclusion: All-cause mortality was similar in live kidney donors and matched non-donor healthy controls with similar health status and socioeconomic status in the Korean population.

Keywords: End-stage kidney disease, Living donors, Mortality, Risk assessment, Prognosis

\section{Introduction}

Kidney transplantation from living donors is the best treatment option for end-stage kidney disease (ESKD). Even considering graft and patient survival, living donor kidney transplantation has shown superior outcomes for recipients compared to kidney transplantation from deceased donors [1]. However, living donor kidney transplantation requires

Received: February 21, 2021; Revised: June 6, 2021; Accepted: June 21, 2021

Correspondence: Hajeong Lee

Department of Internal Medicine, Seoul National University Hospital, 101 Daehak-ro, Jongno-gu, Seoul 03080, Republic of Korea.

E-mail:mdhjlee@gmail.com

ORCID: https://orcid.org/0000-0002-1873-1587

Eunjeong Kang and Sehoon Park contributed equally to this work.

Copyright (C) 2022 by The Korean Society of Nephrology

(a) This is an Open Access article distributed under the terms of the Creative Commons Attribution Non-Commercial and No Derivatives License (http:// creativecommons.org/licenses/by-nc-nd/4.0/) which permits unrestricted non-commercial use, distribution of the material without any modifications, and reproduction in any medium, provided the original works properly cited. 
meticulous considerations regarding various medical, ethical, and moral issues. Considering the complex circumstances surrounding kidney transplantation, living donors might not consider adequately the long-term medical risks of nephrectomy. Physicians managing live kidney donors should recognize these risks and help donors to make reasonable decisions based on scientific evidence.

Long-term safety issues regarding kidney function recovery, quality of life, and the risk of progression to ESKD and all-cause mortality remain controversial. Studies from the United States [2], Canada [3], and South Korea [4] using matched non-donor comparators demonstrated that live kidney donors had similar mortality risks to individuals with a similar baseline health status. However, a Norwegian study reported higher mortality risk for live kidney donors compared with matched controls. A recent meta-analysis showed the absence of a definite increase in the risk of mortality [5]. The estimates of the long-term effects of donor nephrectomy regarding ESKD increased by 8 to 11 times in recent data from a large cohort $[1,6,7]$.

These controversial results are attributed partially to incomplete follow-up medical records for live kidney donors, leading to difficulties in ascertaining long-term medical complications [8]. In addition, selecting individuals who have similar health statuses to donors is crucial for valid comparisons. Pre-existing studies used various comparators, including the general population [5,9-12], non-donating siblings $[13,14]$, or healthy volunteers $[5,15,16]$. Living donors are selected only after careful evaluation that confirms a health status that satisfies the donor criteria, indicating that living donors are healthier than the general population [4]. Since they are highly likely to share the inheritance of kidney disease, hypertension, and diabetes, the effect of donor nephrectomy alone might not be identified when comparing non-sibling donors $[13,17]$. Moreover, most of the above studies adopted controls from population-based administrative data or self-questionnaires instead of objective medical records. It is important to establish healthy non-donor comparators to determine the risks of kidney donation.

Recently, we showed that the mortality risk of Korean live kidney donors did not exceed that of non-donor comparators with similar health status [4]. This previous study was limited by its single-center design; thus, in the current study, we aimed to clarify the long-term risk of all-cause mortality in live kidney donors and healthy non-donor controls from seven national university-affiliated medical centers in South Korea. We linked the medical records of the donors and controls to the National Health Insurance Service (NHIS) database of South Korea to include the nationwide occurrence of mortality.

\section{Methods}

\section{Ethical approval}

This study was approved by the Institutional Review Board (IRB) of each participating clinical center as follows: Seoul National University Hospital (No. H-1903-116-1019), Seoul National University Bundang Hospital (No. B-1905/540402), Seoul National Hospital Boramae Medical Center (No. 20190422/30-2019-28/053), Jeonbuk National University Hospital (No. CUH 2019-05-068), Chonnam National University Hospital (No. CNUH-2019-163), Kyungpook National University Hospital (No. 2019-04-014-001), and Pusan National University Hospital (No. H-1905-018-079). The IRBs waived the requirement for informed consent as the study analyzed an anonymous database provided by the NHIS of South Korea. The study was consistent with the principles of the Declaration of Istanbul.

\section{Study setting}

This study was a retrospective multicenter study performed in seven national university-affiliated hospitals in South Korea. The study included living kidney transplantation donors and a control group that included people who voluntarily received general health screenings. Donors received routine cardiac screenings (e.g., electrocardiogram) before their kidney donations. In addition to the basic analysis, further matching was performed to secure compatibility between the two groups. Baseline information was collected by electronic medical record review in each center to provide more detailed information compared to using the claims database. Further, outcome details were collected from the national claims database of the NHIS of Korea, which generally provides health insurance for all Koreans. The outcome details included both intra-center outcomes and all the outcomes that occurred within South Korea. 


\section{Study population}

The donor group included live kidney donors from 2003 to 2016. The matched healthy non-donor control group included subjects who received general health screenings in one of the hospitals during the same period $[4,18,19]$. As the control group included individuals with various medical conditions, we initially excluded those who overlapped with the donor group and who had an estimated glomerular filtration rate (eGFR) less than $50 \mathrm{~mL} / \mathrm{min} / 1.73 \mathrm{~m}^{2}$. We additionally excluded the study subjects, both donors and controls, according to data availability, including those without identifiable follow-up data in the claims database and those without information regarding important covariates such as age, sex, diabetes mellitus, hypertension, body mass index, eGFR, or dipstick urine results.

\section{Ascertainment of outcomes}

The primary study outcome was all-cause mortality. The NHIS database includes nationwide mortality information through issued death certificates. Mortality outcomes were identified through December 31, 2018.

\section{Data collection}

The baseline covariates were collected from electronic medical records and comprised age, sex, body mass index, history of diabetes mellitus, hypertension, serum creatinine level, eGFR based on the Chronic Kidney Disease Epidemiology Collaboration (CKD-EPI) method [20,21], dipstick albuminuria results, baseline systolic and diastolic blood pressures, and levels of plasma hemoglobin and serum uric acid. In addition, we obtained information on economic status and area of residence from the NHIS database as the socioeconomic status variables; these two variables might affect long-term individual and kidney outcomes. Economic status represented by wealth percentile was collected and stratified based on the health insurance fee in the year of baseline assessment. The information was stratified into aided (the poorest group receiving insurance aid from the government) and quartile (1st-25th, 26th-50th, 51st-75th, and 76th-100th percentile) groups. Areas of residence were stratified as rural or urban, including the capital and metropolitan cities, based on the individual's registered address in the NHIS system. We collected events regarding progression to ESKD through claims information, which issues specific codes for ESKD, as the status receives additional insurance coverage in Korea.

\section{Matching process}

In addition to the basic analysis, we additionally conducted an analysis with a matched dataset. We used direct matching based on the following variables: age (allowing intervals of \pm 5 years and $\geq 60$ years or not), sex, era (allowing intervals of \pm 3 years), body mass index ( $\geq 25 \mathrm{~kg} / \mathrm{m}^{2}$ or not according to the Korean obesity guidelines [22]), presence of prior diabetes mellitus and hypertension, eGFR (allowing intervals of $\pm 10 \mathrm{~mL} / \mathrm{min} / 1.73 \mathrm{~m}^{2}$ ), and presence of dipstick albuminuria (negative/trace or $\geq 1+$ ). Subjects without a match were excluded from the analysis of the matched datasets. Although socioeconomic status was considered initially as a possible matching variable, because of the very large differences in the distribution of status, the variable instead was included as a covariate in the multivariable model. To assess the potential bias resulting from the matching process, we performed bootstrap matching 1,000 times and investigated the distribution of $p$-values and effect sizes in the multivariable-adjusted model.

\section{Statistical analysis}

Categorical variables are presented as number (\%) and continuous variables are presented as median (interquartile range [IQR]). Univariable, age- and sex-adjusted, and multivariable Cox regression models were constructed. The multivariable model included baseline age, sex, time period (2003-2008, 2009-2012, 2013-2016), history of diabetes mellitus or hypertension, body mass index $\left(\geq 25 \mathrm{~kg} / \mathrm{m}^{2}\right.$ or not), dipstick albuminuria (negative/trace or $\geq 1+$ ), baseline eGFR, economic status, and region of residence. The results before and after the matching process are presented. Additional analyses to identify the risk factors for all-cause mortality were conducted, and the analyses included all the donors from 2003 to 2016. In the analyses, all collected variables were investigated for a significant association with progression to ESKD or all-cause mortality. Age ( $\geq 60$ years or less) and eGFR ( $\geq 80 \mathrm{~mL} / \mathrm{min} / 1.73 \mathrm{~m}^{2}$ or less) were recategorized according to the suggested definition for the 
extended criteria donor. In the risk factor analysis, univariable and multivariable logistic regression analyses were employed, and in the multivariable model, the backward-elimination method was used to identify significant risk factors for adverse study outcomes. There was no missing information in the regression models. All statistical analyses were performed using R version 3.6.2 (R Foundation for Statistical Computing, Vienna, Austria), and two-sided p-values of $<0.05$ were considered to indicate statistical significance.

\section{Results}

\section{Study population}

We collected 3,456 donor cases from electronic medical record reviews in the study hospitals (Fig. 1). Among them, 1,578 cases underwent their donations at times other than 2003 to 2016 and thus were excluded. Among the remaining 1,878 donors and 78,115 healthy controls from 2003 to 2016, 1,751 donors and 71,903 healthy controls were included in this study after applying the exclusion criteria. As we could not find exactly matched controls for 50 of the donors, the matched data set included 1,701 donors and the same number of healthy controls.

\section{Characteristics of the study population}

The baseline characteristics before and after the matching process involving all the subjects are presented in Table 1 and Supplementary Table 1 (available online). Before matching, the donors had a relatively lower median age, in addition to lower proportions of male sex, obesity, diabetes mellitus, hypertension, and dipstick albuminuria. They also had higher eGFR values. Regarding economic status or region of residence, the controls had higher wealth percentiles and lived more in urban areas. After matching, the imbalance in the matched variables was no longer observed; however, the matched controls had higher wealth percentiles and higher proportions of urban residence. The prevalence of hypertension was $16.6 \%$ and that of diabetes was $2.7 \%$. There were $1.6 \%$ of matched donors/controls who had dipstick albuminuria.

Before and after matching, no donors progressed to ESKD in the study population. In contrast, 128 healthy controls progressed to ESKD before matching, but none of the controls had ESKD events after matching.

\section{Risks of all-cause mortality}

Before matching, we identified 24 mortality events (incidence rate, 1.64/1,000 person-years; average age at death, $55.1 \pm$ 15.6 years old; average time from index date to death, $6.6 \pm$ 4.5 years) in the donors and 2,533 mortality events (incidence rate, 3.04/1,000 person-years; average age at death, $62.8 \pm$ 11.6 years old; average time from index date to death, $7.8 \pm 4.1$ years) in the healthy controls during the median follow-up duration of 12.5 years (IQR, 9.4-14.4 years) (Table 2). In the univariable model, the donors had a lower risk of all-cause mortality than the controls, but the reverse was noted in the age-sex adjusted model. However, with additional consideration for economic status or regions of residence, the difference in mortality risk was nonsignificant. After matching, while the matched donors had 24 mortality events (incidence rate, 1.68/1,000 person-years; average age at death, $48.5 \pm 14.7$ years old; average time from index date to death, $6.6 \pm 4.5$ years) during the median follow-up duration of 7.7 years (IQR, 4.8-11.8 years), the matched control group had 12 death cases (incidence rate, 0.83/1,000 person-years; average age at death, $49.8 \pm 14.2$ years old; average time from index date to death, $6.1 \pm 4.4$ years) during the median follow-up duration of 7.8 years (IQR, 5.0-12.0 years). The survival analysis showed that the difference in risk of mortality was nonsignificant after consideration for region of residence and economic status, similar to the results before the matching process. When we performed bootstrapping for matching (Table 3), the most (99.4\%) adjusted multivariable model yielded nonsignificant results.

\section{Risk factors for all-cause mortality in living donors}

Regarding risk factors, among the 1,751 donors, including the total cases before matching, from 2003 to 2016, age $\geq$ 60 years was a risk factor for all-cause mortality in the univariable analysis, and it was the only variable that remained after the backward-elimination method (Table 4). Other variables were not significantly associated with the risk of mortality in the donors. 


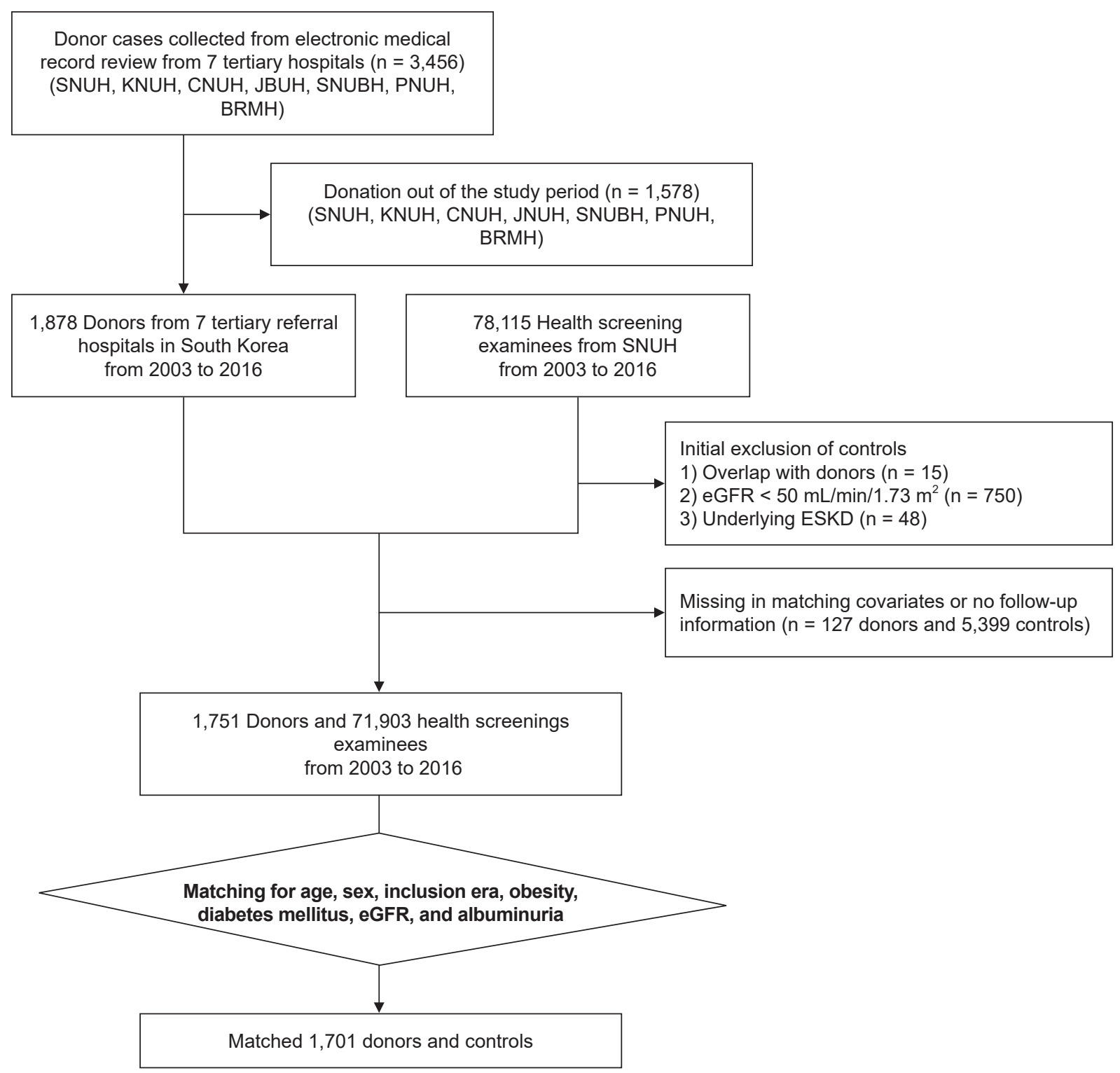

Figure 1. Flow chart showing inclusion and exclusion of kidney donors and non-donor controls. BRMH, Seoul National University Hospital Boramae Medical Center; CNUH, Chonnam National University Hospital; eGFR, estimated glomerular filtration rate; ESKD, end-stage kidney disease; JBUH, Jeonbuk National University Hospital; KNUH, Kyungpook National University Hospital; PNUH, Pusan National University Hospital; SNUBH, Seoul National University Bundang Hospital; SNUH, Seoul National University Hospital.

\section{Discussion}

In this multicenter observational study in South Korea, we identified that the prognosis of live kidney donors was comparable to that of healthy individuals when socioeconomic status was considered. This study suggests that, in this Korean population, the survival of living donors is generally similar to that of healthy individuals.
Because living donors donate kidneys despite perioperative risks and surgical complications, it is essential to provide accurate information regarding the long-term risks and safety of this procedure. However, most live kidney donor studies have been conducted in the Western population $[1-3,6,23,24]$, with a lack of data for Asians. As the proportion of live kidney transplantations is not only substantial but also gradually increasing in South Korea [25], this study 
Table 1. Baseline characteristics

\begin{tabular}{|c|c|c|c|}
\hline \multirow{2}{*}{ Characteristic } & \multicolumn{3}{|c|}{ After matching } \\
\hline & Donor $(n=1,701)$ & Control $(n=1,701)$ & p-value \\
\hline \multicolumn{4}{|l|}{ Matched variable } \\
\hline $\operatorname{Age}^{a}(y r)$ & $44.0(35.0-52.0)$ & $44.0(36.0-52.0)$ & 0.64 \\
\hline$\geq 60$ & $107(6.3)$ & $107(6.3)$ & $>0.99$ \\
\hline $\operatorname{Sex}^{\mathrm{a}}$ & & & $>0.99$ \\
\hline Male & $792(46.6)$ & $792(46.6)$ & \\
\hline Female & 909 (53.4) & 909 (53.4) & \\
\hline Body mass index $\left(\mathrm{kg} / \mathrm{m}^{2}\right)$ & $23.4(21.6-25.4)$ & $23.1(21.0-25.4)$ & 0.002 \\
\hline$\geq 25^{\mathrm{a}}$ & $512(30.1)$ & $512(30.1)$ & $>0.99$ \\
\hline \multicolumn{4}{|l|}{ Underlying disease } \\
\hline Diabetes mellitus $^{a}$ & $46(2.7)$ & $46(2.7)$ & $>0.99$ \\
\hline Hypertension $^{a}$ & $283(16.6)$ & $283(16.6)$ & $>0.99$ \\
\hline Creatinine $(\mathrm{mg} / \mathrm{dL})$ & $0.8(0.7-0.9)$ & $0.8(0.7-0.9)$ & 0.51 \\
\hline eGFRa (mL/min/1.73 m²) & $100.3(89.0-108.9)$ & $100.1(89.1-108.5)$ & 0.46 \\
\hline Dipstick albuminuria $^{a}(\geq 1+)$ & $28(1.6)$ & $28(1.6)$ & $>0.99$ \\
\hline \multicolumn{4}{|l|}{ Unmatched variable } \\
\hline Systolic blood pressure (mmHg) & $120.0(110.0-130.0)$ & $118.0(109.0-129.0)$ & 0.39 \\
\hline Diastolic blood pressure (mmHg) & $73.0(67.0-80.0)$ & $72.0(65.0-79.0)$ & 0.002 \\
\hline Hemoglobin (g/dL) & $13.7(12.7-15.0)$ & $14.2(13.1-15.4)$ & $<0.001$ \\
\hline Uric acid (mg/dL) & $4.8(4.0-5.9)$ & $5.1(4.2-6.2)$ & $<0.001$ \\
\hline Wealth percentile & & & $<0.001$ \\
\hline 0 (aided) & $68(4.0)$ & $2(0.1)$ & \\
\hline 1st-25th & $332(19.5)$ & $174(10.2)$ & \\
\hline 26th-50th & $378(22.2)$ & $198(11.6)$ & \\
\hline 51st-75th & $426(25.0)$ & $369(21.7)$ & \\
\hline 76th-100th & $497(29.2)$ & $958(56.3)$ & \\
\hline Place of residence & & & $<0.001$ \\
\hline Rural & $588(34.6)$ & $269(15.8)$ & \\
\hline Urban & $1,113(65.4)$ & $1,432(84.2)$ & \\
\hline
\end{tabular}

Data are expressed as median (interquartile range) or number (\%).

eGFR, estimated glomerular filtration rate.

${ }^{a}$ Directly matched variables.

is important to clarify the long-term mortality of living donors, especially in the Korean population. We compared long-term all-cause mortality outcomes of live kidney donors using a well-characterized non-donor control group with similar health status and found it comparable all-cause mortality in the two groups. This was shown repeatedly in the sensitivity analysis using the bootstrap method.

There are some difficulties in conducting studies for live kidney donors. First, because live kidney donors are determined after detailed health screening tests, it is important to select a comparative group with similar health status. Additionally, because living donors are healthy individuals, long-term follow-up is required to observe notable clinical outcomes such as ESKD and mortality. Although several studies have compared live kidney donors with matched comparison groups, some studies [2-4] have reported that donors had similar mortality risks compared to matched controls, but a large-scale Norwegian study [1] showed a higher mortality risk for donors. Thus, there are some controversies to date regarding the long-term risks of live kidney donors (Table 5).

In a single-center study in South Korea by Kim et al. [4], which was the first report to include long-term mortality data in an Asian population, the mean follow-up duration was 12 years, and the risks of all-cause mortality were similar between live kidney donors and the matched 
Table 2. Risk of all-cause mortality in the donors and controls from 2003 to 2016

\begin{tabular}{|c|c|c|c|c|c|c|c|}
\hline \multirow{2}{*}{ Matching and group } & \multirow{2}{*}{$\begin{array}{c}\text { Outcome/ } \\
\text { subjects (n) }\end{array}$} & \multicolumn{2}{|c|}{ Univariable model } & \multicolumn{2}{|c|}{ Age-sex adjusted model } & \multicolumn{2}{|c|}{ Multivariable model $^{a}$} \\
\hline & & $\mathrm{HR}(95 \% \mathrm{Cl})$ & p-value & Adjusted HR (95\% Cl) & p-value & Adjusted HR (95\% Cl) & p-value \\
\hline \multicolumn{8}{|l|}{ Before matching } \\
\hline Donor & $24 / 1,751$ & $0.60(0.40-0.90)$ & 0.01 & $1.68(1.12-2.52)$ & 0.01 & $1.41(0.94-2.13)$ & 0.10 \\
\hline Control & $2,533 / 71,903$ & Reference & & Reference & & Reference & \\
\hline \multicolumn{8}{|l|}{ After matching } \\
\hline Donor & $24 / 1,701$ & $2.01(1.01-4.03)$ & 0.05 & $2.00(1.00-4.00)$ & 0.05 & $1.82(0.87-3.80)$ & 0.11 \\
\hline Control & $12 / 1,701$ & Reference & & Reference & & & \\
\hline
\end{tabular}

$\mathrm{Cl}$, confidence interval; $\mathrm{HR}$, hazard ratio.

${ }^{a}$ Adjusted for age, sex, era, history of diabetes mellitus, hypertension, body mass index, dipstick albuminuria, baseline estimated glomerular filtration rate, economic status, and region of residence (urban or rural).

Table 3. Results bootstrapped for 1,000 iterations of the matching process with the matched population included from 2003 to 2016

\begin{tabular}{lccccc}
\hline Variable & No. of $p<0.05$ & $p$-value (IQR) & HR (IQR) & No. of HR $>1$ & No. of HR $>2$ \\
\hline Univariable model & 526 & $0.05(0.03-0.10)$ & $2.01(1.73-2.20)$ & 1,000 & 524 \\
Age- and sex-adjusted model & 424 & $0.05(0.03-0.11)$ & $2.00(1.72-2.18)$ & 1,000 & 371 \\
Multivariable model & 60 & $0.13(0.08-0.20)$ & $1.76(1.59-1.94)$ & 1,000 & 181 \\
\hline
\end{tabular}

$\mathrm{HR}$, hazard ratio; IQR, interquartile range.

comparison group. However, their study was limited by its single-center nature, the enrollment of $7.7 \%$ of patients with a follow-up period of $<1$ year, and evaluation of allcause mortality alone without considering ESKD. Although our study used a similar comparator group as this previous study, there are several differences between the two studies. Our current study was a multicenter study that included seven national universities across South Korea and acquired ample detailed clinical outcome data by linking electrical medical records and administrative data from NHIS. The current study, which complements the previous study, showed that the long-term rates of all-cause mortality of donors and non-donors were similar, particularly when the baseline socioeconomic status was considered. Moreover, it is noteworthy that the absolute numbers of ESKD and mortality events of live kidney donors are small in South Korea, indicating that live kidney donation is a safe procedure in this population.

Considering that more diabetic and hypertensive patients were included than in other studies, the absolute outcome risk in this Korean population might be low (Table 5). The exact mechanism of this phenomenon is unknown, but Asians are considered to have lower metabolic risks for adverse health outcomes such as obesity [26] and diabetes mellitus $[27,28]$ compared to Western populations.

An important finding in the present study is that socio- economic status was the major confounding factor for mortality outcomes $[29,30]$. Differences in socioeconomic status and area of residence were observed between the matched donors and the non-donor healthy controls. Because these differences were notable, these two variables could not be included as matching variables. Because the matched non-donor healthy controls included in this study self-funded their voluntary medical check-ups, it is likely that their socioeconomic status and interest in health were higher than those of the donors. Thus, we conducted an analysis including socioeconomic status and region of residence as covariates. The significant difference in mortality was not observed in the multivariable analysis when both variables were included as covariates. This means that socioeconomic status might be a major confounder regarding the risk of long-term donor mortality, which is evident in the general population of those with chronic kidney disease $[31,32]$. Further studies are needed to clarify the impact of socioeconomic status on the long-term outcomes of kidney donors owing to the limited evidence regarding this subject. Moreover, our study suggests the importance of considering socioeconomic status when comparing the prognoses of living donors to those of healthy individuals.

There are some limitations to this study. First, the hospitals included in this study might have different donor management programs and exclusion criteria according to 
Table 4. Risk factors for all-cause mortality in all donors during 2003 to 2016

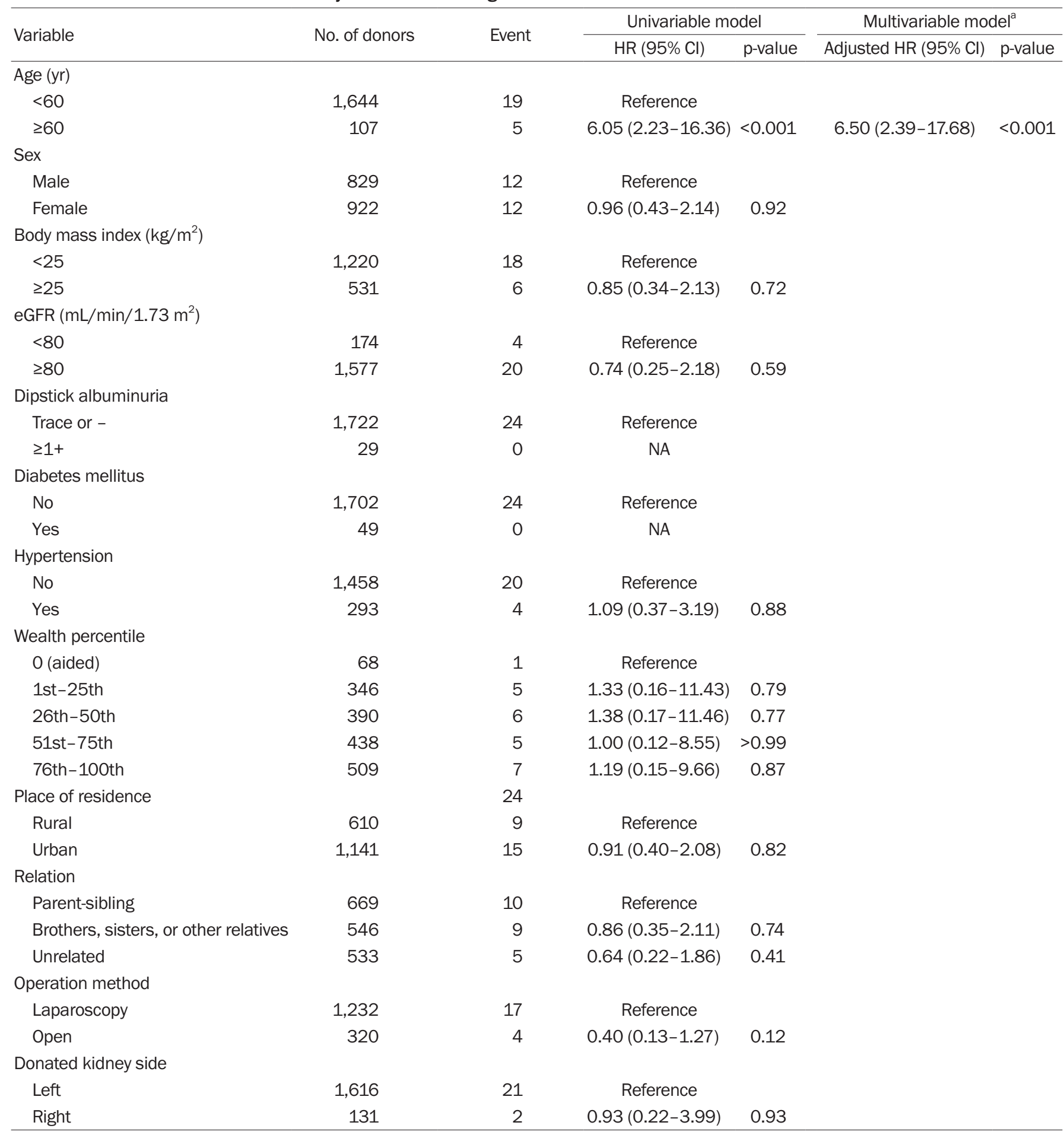

The effect sizes in the multivariable model were different from those in the univariable model, although a single variable (age $\geq 60$ years) remained after backward elimination because the multivariable analysis was performed with 1,744 individuals due to missing data for the considered variables.

$\mathrm{Cl}$, confidence interval; eGFR, estimated glomerular filtration rate; HR, hazard ratio; NA, not applicable.

${ }^{\mathrm{a}}$ By backward-elimination method. 
Table 5. Comparison of baseline characteristics and follow-up durations with those of previous retrospective studies on long-term risk after living kidney donation

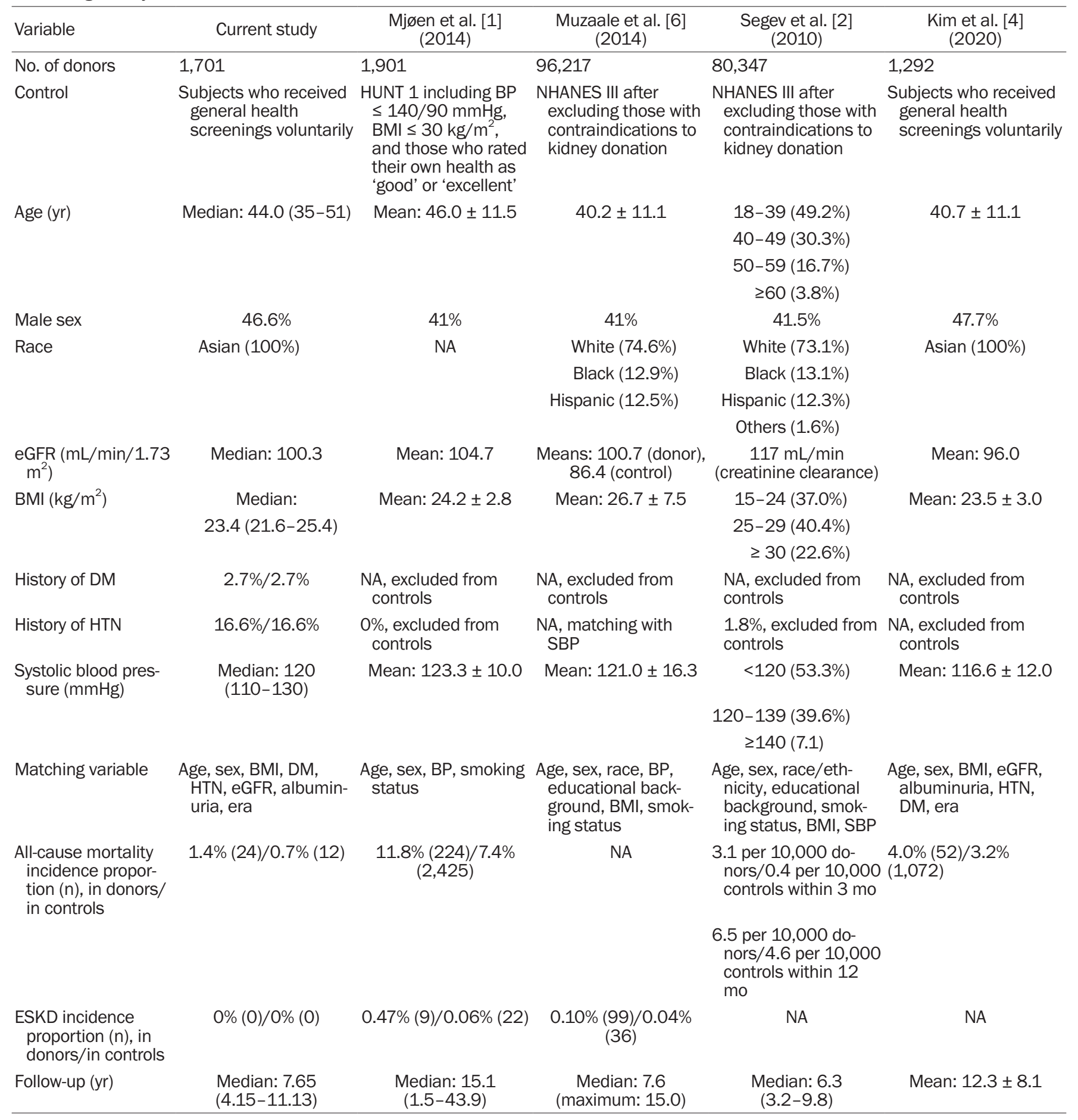

BMI, body mass index; BP, blood pressure; DM, diabetes mellitus; eGFR, estimated glomerular filtration rate; ESKD, end-stage kidney disease; HTN, hypertension; HUNT, Health Study of Nord-Trøndelag; NA, not applicable; NHANES III, the third National Health and Nutritional Examination Survey; SBP, systolic BP. 
underlying disease, diabetes, donor age, and willingness to transplant of donors. Second, the absolute number of deaths was small because the donors were healthy individuals. However, this reflects the small absolute risk of adverse outcomes in living donors in South Korea. Additionally, the exact cause of death was unknown in this population, and the mortality events of living donors might not be associated with the donation itself. Moreover, donor data have been collected in electronic medical records since the 1980s, and the linked data from NHIS were established in 2003. Therefore, patients who had a clinical outcome before 2003 could not be detected. Last, the limited follow-up duration due to unavailability of data prevented us from investigating the long-term prognosis of living donors.

In conclusion, the risk of all-cause mortality was comparable between live kidney donors and matched non-donor healthy controls with similar health status in an Asian population after consideration of several clinicodemographic characteristics. Considering the relatively low absolute risk of mortality or ESKD, live kidney donation should not be discouraged. However, potential donors should be informed of the long-term risks, and only those who accept the risks should undergo donor nephrectomy. The present study adds to the evidence regarding medical considerations in live kidney donation. Additional long-term studies including larger numbers of donors and non-donor controls are needed to determine the risks of live kidney donation, including ESKD.

\section{Additional information}

${ }^{1}$ Department of Internal Medicine, Ewha Womans University Seoul Hospital, Ewha Womans University College of Medicine, Seoul, Republic of Korea

${ }^{2}$ Department of Biomedical Science, Seoul National University College of Medicine, Seoul, Republic of Korea

${ }^{3}$ Department of Biostatistics, Korea University College of Medicine, Seoul, Republic of Korea

${ }^{4}$ Department of Internal Medicine, Keimyung University Dongsan Hospital, Daegu, Republic of Korea

${ }^{5}$ Department of Statistics, Keimyung University, Daegu, Republic of Korea

${ }^{6}$ Transdisciplinary Department of Medicine \& Advanced Technology, Seoul National University Hospital, Seoul, Republic of Korea

${ }^{7}$ Division of Healthcare Technology Assessment Research, National Evidence-based Healthcare Collaborating Agency, Seoul, Republic of Korea

${ }^{8}$ Department of Internal Medicine, Hallym University Hangang Sacred Heart Hospital, Seoul, Republic of Korea
${ }^{9}$ Department of Internal Medicine, Kyungpook National University Hospital, Daegu, Republic of Korea

${ }^{10}$ Department of Internal Medicine, Seoul National University Boramae Medical Center, Seoul, Republic of Korea

${ }^{11}$ Department of Internal Medicine, Jeonbuk National University Hospital, Jeonju, Republic of Korea

${ }^{12}$ Department of Internal Medicine, Chonnam National University Hospital, Gwangju, Republic of Korea

${ }^{13}$ Department of Family Medicine, Seoul National University Hospital, Seoul, Republic of Korea

${ }^{14}$ Department of Internal Medicine, Seoul National University Bundang Hospital, Seongnam, Republic of Korea

${ }^{15}$ Department of Internal Medicine, Seoul National University Hospital, Seoul, Republic of Korea

\section{Conflicts of interest}

All authors have no conflicts of interest to declare.

\section{Funding}

This study was supported by the National Evidence Collaborating Agency (project number NECA-A-20-005). The funder had no role in performing the study, and the study was independently performed by the authors.

\section{Authors' contributions}

Conceptualization: EK, SP, HL

Investigation: EK, YK, HJK, MH, JHC, JPL, SL, SWK, SMP, DWC, HJC, YSK, YCK, IC, HL

Formal analysis: EK, SP, JP, MP, KK

Funding acquisition: HL

Writing-original draft: EK, SP

Writing-review \& editing: HL

All authors read and approved the final manuscript.

\section{ORCID}

Eunjeong Kang, https://orcid.org/0000-0002-2191-2784

Sehoon Park, https://orcid.org/0000-0002-4221-2453

Jina Park, https://orcid.org/0000-0002-7410-1985

Yaerim Kim, https://orcid.org/0000-0003-1596-1528

Minsu Park, https://orcid.org/0000-0002-0624-5215

Kwangsoo Kim, https://orcid.org/0000-0002-4586-5062

Hyo Jeong Kim, https://orcid.org/0000-0002-5618-0042

Miyeun Han, https://orcid.org/0000-0001-7304-2496

Jang-Hee Cho, https://orcid.org/0000-0002-7031-5214 
Jung Pyo Lee, https://orcid.org/0000-0002-4714-1260

Sik Lee, https://orcid.org/0000-0002-9043-8166

Soo Wan Kim, https://orcid.org/0000-0002-3540-9004

Sang Min Park, https://orcid.org/0000-0002-7498-4829

Dong-Wan Chae, https://orcid.org/0000-0001-9401-892X

Ho Jun Chin, https://orcid.org/0000-0003-1185-2631

Yong Chul Kim, https://orcid.org/0000-0003-3215-8681

Yon Su Kim, https://orcid.org/0000-0003-3091-2388

Insun Choi, https://orcid.org/0000-0003-2091-5964

Hajeong Lee, https://orcid.org/0000-0002-1873-1587

\section{References}

1. Mjøen G, Hallan S, Hartmann A, et al. Long-term risks for kidney donors. Kidney Int 2014;86:162-167.

2. Segev DL, Muzaale AD, Caffo BS, et al. Perioperative mortality and long-term survival following live kidney donation. JAMA 2010;303:959-966.

3. Garg AX, Meirambayeva A, Huang A, et al. Cardiovascular disease in kidney donors: matched cohort study. BMJ 2012; 344:e1203.

4. Kim Y, Yu MY, Yoo KD, et al. Long-term mortality risks among living kidney donors in Korea. Am J Kidney Dis 2020;75:919-925.

5. O'Keeffe LM, Ramond A, Oliver-Williams C, et al. Mid- and long-term health risks in living kidney donors: a systematic review and meta-analysis. Ann Intern Med 2018;168:276-284.

6. Muzaale AD, Massie AB, Wang MC, et al. Risk of end-stage renal disease following live kidney donation. JAMA 2014;311:579-586.

7. Maggiore U, Budde K, Heemann U, et al. Long-term risks of kidney living donation: review and position paper by the ERA-EDTA DESCARTES working group. Nephrol Dial Transplant 2017; 32:216-223.

8. Muzaale AD, Massie AB, Segev DL. Concerns about the longterm safety of live kidney donors are justified. Eur J Epidemiol 2017;32:91-93.

9. Mjøen G, Øyen O, Holdaas H, Midtvedt K, Line PD. Morbidity and mortality in 1022 consecutive living donor nephrectomies: benefits of a living donor registry. Transplantation 2009;88:12731279.

10. Fehrman-Ekholm I, Elinder CG, Stenbeck M, Tydén G, Groth CG. Kidney donors live longer. Transplantation 1997;64:976-978.

11. Fournier C, Pallet N, Cherqaoui Z, et al. Very long-term follow-up of living kidney donors. Transpl Int 2012;25:385-390.

12. Kido R, Shibagaki Y, Iwadoh K, et al. How do living kidney donors develop end-stage renal disease? Am J Transplant 2009;9:2514-
2519.

13. Williams SL, Oler J, Jorkasky DK. Long-term renal function in kidney donors: a comparison of donors and their siblings. Ann Intern Med 1986;105:1-8.

14. Najarian JS, Chavers BM, McHugh LE, Matas AJ. 20 years or more of follow-up of living kidney donors. Lancet 1992;340:807-810.

15. Clemens K, Boudville N, Dew MA, et al. The long-term quality of life of living kidney donors: a multicenter cohort study. Am J Transplant 2011;11:463-469.

16. Young A, Hodsman AB, Boudville N, et al. Bone and mineral metabolism and fibroblast growth factor 23 levels after kidney donation. Am J Kidney Dis 2012;59:761-769.

17. Connor SL, Connor WE, Henry H, Sexton G, Keenan EJ. The effects of familial relationships, age, body weight, and diet on blood pressure and the 24 hour urinary excretion of sodium, potassium, and creatinine in men, women, and children of randomly selected families. Circulation 1984;70:76-85.

18. Lee H, Oh SW, Heo NJ, et al. Serum phosphorus as a predictor of low-grade albuminuria in a general population without evidence of chronic kidney disease. Nephrol Dial Transplant 2012;27:2799-2806.

19. Yoo KD, Kang S, Choi Y, et al. Sex, age, and the association of serum phosphorus with all-cause mortality in adults with normal kidney function. Am J Kidney Dis 2016;67:79-88.

20. Levey AS, Stevens LA, Schmid CH, et al. A new equation to estimate glomerular filtration rate. Ann Intern Med 2009;150:604612.

21. Levey AS, Stevens LA. Estimating GFR using the CKD Epidemiology Collaboration (CKD-EPI) creatinine equation: more accurate GFR estimates, lower CKD prevalence estimates, and better risk predictions. Am J Kidney Dis 2010;55:622-627.

22. Seo MH, Lee WY, Kim SS, et al. 2018 Korean Society for the Study of Obesity Guideline for the Management of Obesity in Korea. $J$ Obes Metab Syndr 2019;28:40-45.

23. Garg AX, Prasad GV, Thiessen-Philbrook HR, et al. Cardiovascular disease and hypertension risk in living kidney donors: an analysis of health administrative data in Ontario, Canada. Transplantation 2008;86:399-406.

24. Janki S, Dehghan A, van de Wetering J, et al. Long-term prognosis after kidney donation: a propensity score matched comparison of living donors and non-donors from two population cohorts. Eur J Epidemiol 2020;35:699-707.

25. World Health Organization (WHO). International report on organ donation and transplantation activities executive summary [Internet]. Geneva: WHO; 2017 [cited 2021 Feb 21]. 
Available from: http://www.transplant-observatory.org/ download/2017-activity-data-report/.

26. Wang L, Southerland J, Wang K, et al. Ethnic differences in risk factors for obesity among adults in California, the United States. J Obes 2017;2017:2427483.

27. Whiting DR, Guariguata L, Weil C, Shaw J. IDF diabetes atlas: global estimates of the prevalence of diabetes for 2011 and 2030. Diabetes Res Clin Pract 2011;94:311-321.

28. Karter AJ, Ferrara A, Liu JY, Moffet HH, Ackerson LM, Selby JV. Ethnic disparities in diabetic complications in an insured population. JAMA 2002;287:2519-2527.

29. Pillay-van Wyk V, Bradshaw D. Mortality and socioeconomic status: the vicious cycle between poverty and ill health. Lancet Glob
Health 2017;5:e851-e852.

30. Breeze E, Sloggett A, Fletcher A. Socioeconomic and demographic predictors of mortality and institutional residence among middle aged and older people: results from the Longitudinal Study. J Epidemiol Community Health 1999;53:765-774.

31. Tao S, Zeng X, Liu J, Fu P. Socioeconomic status and mortality among dialysis patients: a systematic review and meta-analysis. Int Urol Nephrol 2019;51:509-518.

32. Meijer M, Kejs AM, Stock C, Bloomfield K, Ejstrud B, Schlattmann P. Population density, socioeconomic environment and all-cause mortality: a multilevel survival analysis of 2.7 million individuals in Denmark. Health Place 2012;18:391-399. 